Bundesgesundheitsbl -

Gesundheitsforsch - Gesundheitsschutz

2001 -44:1153-1161 @ Springer-Verlag 2001

\title{
Nationale und globale Influenza- surveillance als Basis der jährlichen Impfstoffempfehlung
}

\section{Zusammenfassung}

Die Influenza ist noch immer eine der bedeutendsten Infektionskrankheiten, die allein in Deutschland während einer Saison mit ausgeprägter Influenzawelle (z. B. 1998/99) über 10.000 Todesopfer forderte. Mit der Zulassung von Neuraminidaseinhibitoren wurden die therapeutischen Möglichkeiten wesentlich verbessert. Die Immunisierung wird aber nach wie vor die wichtigste Maßnahme zur Bekämpfung der Influenza darstellen. Aufgrund der großen Variabilität der Influenzaviren ist es erforderlich, die Grippeschutzimpfung jährlich zu aktualisieren. Punktmutationen im Genom des Virus führen zu einer sukzessiven Veränderung der viralen Hüllproteine und somit zu einer Antigendrift. Um ein Monitoring und eine rasche Identifizierung von Driftvarianten zu ermöglichen, hat die WHO ein globales Netz zur Überwachung etabliert. Die Basis dieses Netzwerkes bilden 110 nationale Influenzazentren, die die zirkulierenden Influenzaviren isolieren, typisieren und subtypisieren. Neben der Übermittlung von Surveillancedaten besteht die wichtigste Aufgabe darin, die Verwandtschaft der Viren untereinander und mit relevanten Impfstämmen zu untersuchen. Eine Auswahl repräsentativer Stämme wird anschließend den vier regionalen WHO Collaborating Centres für vergleichende Analysen zu Verfügung gestellt. So kann ermittelt werden, welche Varianten zirkulieren oder sich besonders verbreiten. Als Ergebnis der weltweit zirkulierenden Stämme wird von der WHO eine separate Impfstoffempfehlung für die nördliche und die südliche Hemisphäre ausgesprochen.

\section{Schlüsselwörter}

Influenzasurveillance · Influenza A . Influenza B · Typisierung · Subtypisierung

\section{Epidemiologie der Influenza}

Die Influenza - die echte Virusgrippe zählt nach wie vor zu den bedeutendsten Infektionskrankheiten. Das ätiologische Agens dieser Erkrankung sind Influenzaviren, die aufgrund der antigenen Eigenschaften ihrer internen Proteine in die Typen A, B und C eingeteilt werden. Influenza-A- und -B-Viren sind verantwortlich für weltweit auftretende Epidemien. Influenza-C-Viren sind nicht von epidemiologischer Relevanz, denn Erkrankungen durch dieses Virus treten nur sporadisch auf. Das morphologische Charakteristikum der Influenzaviren sind Spike-artige Projektionen an der Oberfläche des Virus, die durch die beiden Oberflächenproteine Hämagglutinin (HA) und Neuraminidase (NA) gebildet werden. Aufgrund der antigenen Eigenschaften dieser Hüllproteine werden Influenza-A-Viren in eine Vielzahl von Subtypen unterteilt. Es sind 15 HAund neun NA-Subtypen bekannt [1].

Das erste Influenzavirus wurde 1933 isoliert. Seit dieser Zeit stehen virologische und serologische Methoden zur Verfügung, die beim Menschen eine intensive Zirkulation der Subtypen $\mathrm{H}_{1} \mathrm{~N} 1$, $\mathrm{H}_{2} \mathrm{~N}_{2}$ und $\mathrm{H}_{3} \mathrm{~N}_{2}$ im 2O. Jahrhundert belegen. Diese Methoden erlaubten auch die retrospektive Klärung der letzten Pandemien des 19. Jahrhunderts. Bereits die Pandemie von 1889 wurde durch den Subtyp $\mathrm{H}_{2}$ und die von 1898 durch den Subtyp $\mathrm{H}_{3}$ verursacht.

Abgesehen vom Menschen haben Influenza-A-Viren ein großes tierisches Reservoire. Bei Schweinen zirkulieren die Subtypen $\mathrm{H}_{1} \mathrm{~N}_{1}$ sowie $\mathrm{H}_{3} \mathrm{~N}_{2}$. Für die Pferdeinfluenza sind die Subtypen $\mathrm{H}_{3} \mathrm{~N} 8$ sowie $\mathrm{H}_{7} \mathrm{~N}_{7}$ verantwortlich. Alle Subtypen der Influenza-A-Viren wurden bei
Vögeln nachgewiesen, die daher als ursprünglicher Wirt der Influenza-A-Viren und als Ursprung der für den Menschen gefährlichen Pandemiestämme angesehen werden. Abgesehen vom plötzlichen Auftreten eines besonders pathogenen Stammes verläuft die Infektion bei den Vögeln im Gegensatz zu Mensch und Säugetier meist asymptomatisch. Influenza-B-Viren werden nur beim Menschen gefunden [1].

Besonders charakteristisch für die Influenzaviren ist ihre große Variabilität. Die Hüllproteine dieser Viren verändern sich kontinuierlich, wodurch es möglich ist, eine zuvor aufgebaute Immunität immer wieder neu zu unterlaufen. Diese sich ständig vollziehende Veränderung der antigenen Eigenschaften der Influenzaviren wird als „Antigendrift" bezeichnet. Die permanent ablaufenden Driftereignisse sind auch die Ursache dafür, dass eine einmalige Immunisierung keine dauerhafte Immunität bieten kann und der Impfstoff ständig aktualisiert werden muss [2]. Ursache für die Antigendrift sind Punktmutationen im Genom. Vollzieht sich die Änderung des Virusgenoms nicht schrittweise, sondern drastisch, spricht man von „Antigenshift“. Bei einer Shift kommt es zum Austausch ganzer Gensegmente. Ursache dafür sind Doppelinfektionen sowohl mit humanen als auch aviären Viren (Vogelviren) und einer während der Virusreplikation stattfindenden „Neusortierung“ (Reassortment) der acht viralen Gensegmente. Die so entstehenden Reassortanten können dann z. B. Oberflächenproteine besitzen, die

Dr. Brunhilde Schweiger

Nationales Referenzzentrum für Influenza, Robert Koch-Institut, Nordufer 20, 13353 Berlin 
Bundesgesundheitsbl -

Gesundheitsforsch - Gesundheitsschutz

2001 · 44:1153-1161 @ Springer-Verlag 2001

\section{B. Schweiger}

Vaccine Recommendations are Based on National and Global Influenza Surveillance

\section{Abstract}

Influenza is still one of the most important infectious diseases. In Germany, excess mortality rates of more than 10,000 deaths were reported during a season with high influenza activity (e.g. 1998/99). Treatment of influenza infections was greatly improved by the availability of neuraminidase inhibitors. Nevertheless, vaccination will remain the most important option for controlling influenza. Changes in the viral surface proteins (antigenic variation), are largely responsible for the epidemiological success of the virus. Such an antigenic drift is caused by accumulations of point mutations in the viral genome. These variations render an individual susceptible to new strains, despite previous immunization or infection by other influenza viruses. Due to the constantly changing composition of influenza viruses in circulation, the influenza vaccine has to be modified every year to match the current viruses. Therefore, detailed knowledge about the circulating strains of influenza viruses is necessary. In order to gather these data, an international network has been created by the WHO. This network consists of 110 national and four global WHO collaborating centres. Data of weekly reports cover influenza activity as well as the number of influenza specimens isolated by type and subtype. The circulating influenza viruses have to be characterized serologically by the national centres, and a selection of strains has to be sent rapidly to the WHO collaborating centres for further strain identification. Results from the global influenza surveillance are reviewed each February and September.

\section{Keywords}

Influenza surveillance · Influenza A . Influenza B · Typing · Subtyping

\section{Originalien und Übersichtsarbeiten}

bei humanen Viren noch nie oder schon vor langer Zeit, z. B. einem Jahrhundert, aufgetreten sind. Gegen solche neuen Viren besteht natürlich keine Immunität in der Bevölkerung. Die Folge sind sich global ausbreitende Epidemien, die als Pandemien bezeichnet werden.

\section{„Besonders charakteristisch für die Influenzaviren ist ihre große Variabilität."}

Das letzte Jahrhundert war durch drei große Pandemien gekennzeichnet. Die schwerste Pandemie mit weltweit etwa 40 Millionen Todesopfern wurde 1918 durch den neu auftretenden InfluenzaA-Subtyp $\mathrm{H}_{1} \mathrm{~N}_{1}$ hervorgerufen. Umfangreiche Analysen sprechen dafür, dass es ein $\mathrm{H}_{1} \mathrm{~N}_{1}$-Vogelvirus geschafft hat, in toto in die Population einzudringen [3, 4, 5] und sich an den Menschen anzupassen. Die Pandemie von 1918/19 ging unter dem Namen „Spanische Grippe“ in die Geschichte ein. Die zweite Pandemie von 1957 („Hongkong-Grippe“) wurde durch den damals neu entstandenen Subtyp $\mathrm{H}_{2} \mathrm{~N}_{2}$ hervorgerufen und die „Asiatische Grippe“ von 1968 durch den dann auftretenden neuen Subtyp $\mathrm{H}_{3} \mathrm{~N}_{2}$. Ursache für beide Pandemien war ein genetisches Reassortment bedingt durch eine Doppelinfektion mit einem humanen und einem Vogel-Influenzavirus. Für die Pandemie 1968/69 war ein $\mathrm{H}_{3} \mathrm{~N}_{2}$-Virus verantwortlich, das aus dem Reassortment eines aviären $\mathrm{H}_{3}$-Virus mit dem von 1957 bis 1968 zirkulierenden Subtyp
$\mathrm{H}_{2} \mathrm{~N}_{2}$ hervorgegangen ist. Die Neuraminidase des Pandemievirus von 1968/69 war mit der Neuraminidase des Vorläufer-Subtyps $\mathrm{H}_{2} \mathrm{~N}_{2}$ verwandt [6]. Das Auftreten eines neuen Subtyps führte immer zum Verschwinden des bis dahin zirkulierenden Influenza-A-Subtyps. Eine Ausnahme bildete die Epidemie 1977. In diesem Jahr tauchte der Subtyp H1N1 wieder auf, der eine enge Verwandtschaft mit den Viren aufwies, die bis 1957 zirkulierten [7]. Seit 1977 findet man daher Influenza-A-Viren sowohl vom Subtyp $\mathrm{H}_{3} \mathrm{~N}_{2}$ als auch vom Subtyp $\mathrm{H}_{1} \mathrm{~N}_{1}$ und ebenso Influenza-B-Viren. Von Jahr zu Jahr kann es sehr unterschiedlich sein, welcher Typ bzw. Subtyp überwiegend am Infektionsgeschehen beteiligt ist.

\section{Klinik der Influenza}

Die Influenza ist eine akute Erkrankung der Atemwege. Zu den charakteristischen Symptomen zählt der akute Krankheitsbeginn mit plötzlich auftretendem Fieber, Kopf-, Muskel- und Gliederschmerzen sowie einem trockenen Husten. Diese Symptomatik ist bei der überwiegenden Zahl der Erkrankungen zu finden. Ein vergleichsweise geringerer Anteil der nachgewiesenen Influenzavirusinfektionen kann auch einen milderen Verlauf nehmen [8]. Die Influenza ist keinesfalls eine banale Erkrankung. Menschen mit einem intakten Immunsystem haben die Krankheit meist in zwei Wochen überwunden, berichten jedoch oft über eine sich daran anschließende wochenlange Rekonvaleszenz

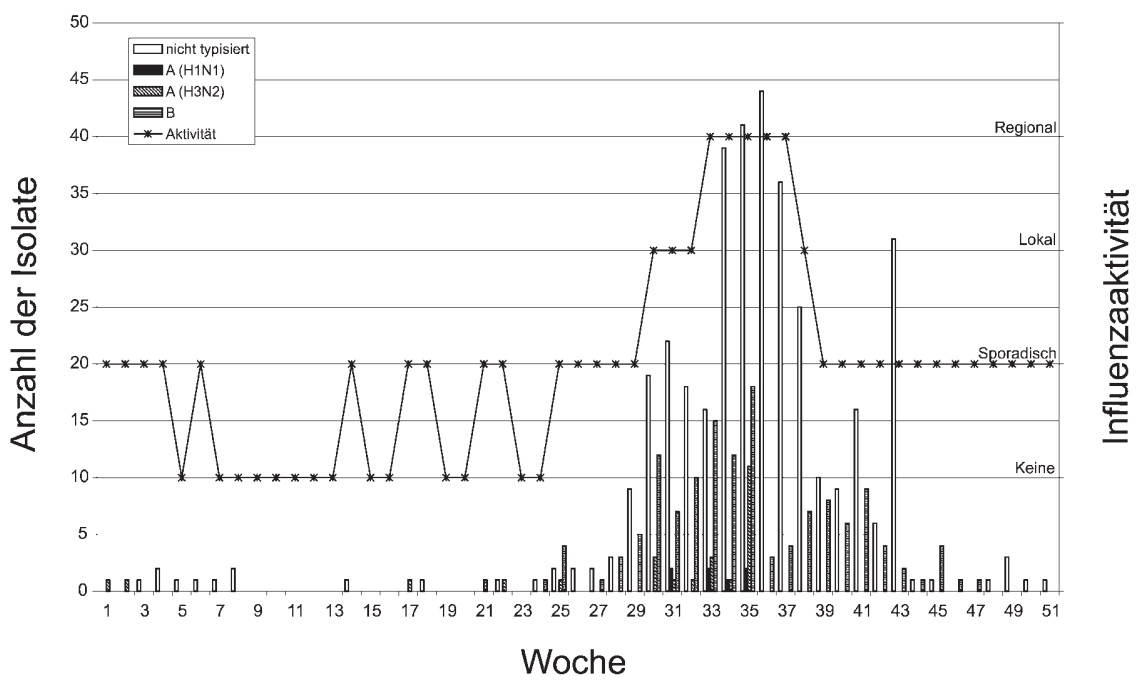

Abb. $1 \Delta$ Influenzasurveillance in Australien im Jahr 2000 (Quelle: FluNet) 


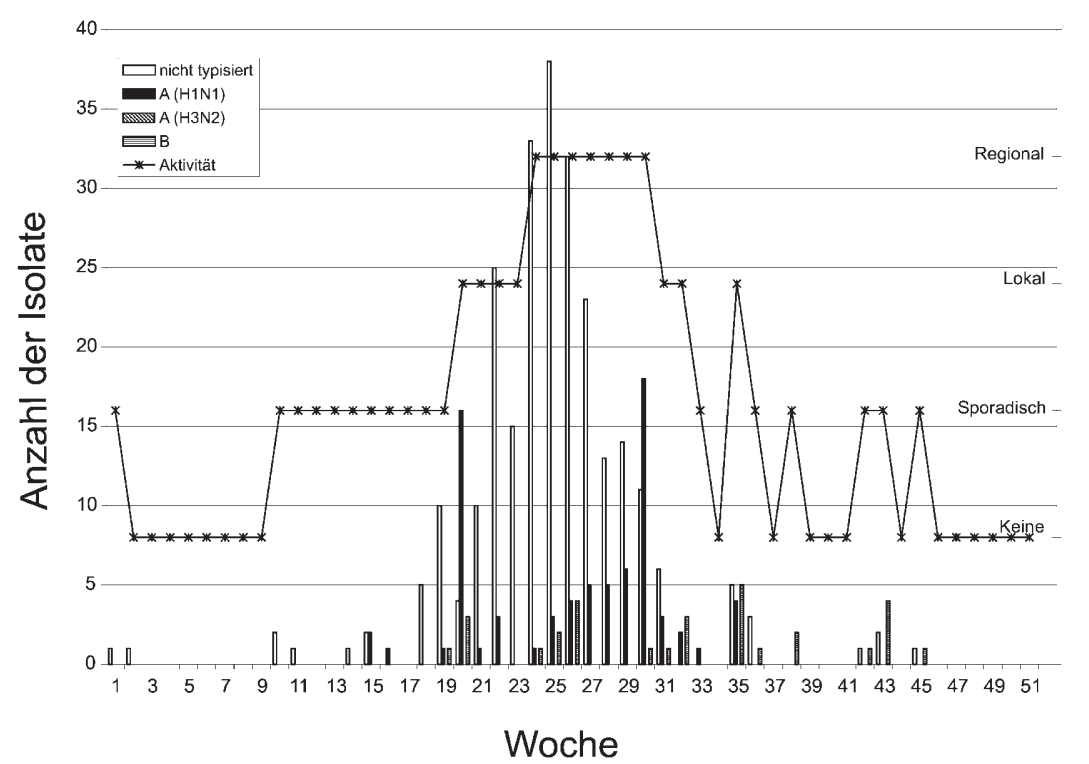

Abb.2 Influenzasurveillance in Argentinien im Jahr 2000 (Quelle: FluNet)

gische Daten gesammelt und ausgewertet. Dieses im Laufe der Jahrzehnte immer besser ausgebaute Surveillance-System basiert auf der Zusammenarbeit mit nationalen Influenzazentren und, soweit in den einzelnen Ländern verfügbar, nationalen Surveillance-Systemen. Die Basis des WHO-Netzwerkes (FluNet) bilden zurzeit 110 nationale Influenzazentren in 82 Ländern und vier WHO Collaborating Centres for Reference and Research in Australien, Japan, Großbritannien und den USA [17]. Ziel dieser globalen Surveillance ist die schnelle Erfassung und Beobachtung neu auftauchender Driftvarianten und Subtypen. Infektionen des Menschen mit aviären Stämmen wie dem Subtyp $\mathrm{H}_{5} \mathrm{~N}_{1}$ im Jahre 1997 [18] oder dem Subtyp H9N2 im Jahr 1999 [19] zeigen, dass die Gefahr einer Influenzapandemie nach wie vor besteht.

(postgrippale Asthenie). Zu den gefürchteten Komplikationen einer Influenza zählen Pneumonie [9,10], Myokarditis, Perikarditis [11] und Enzephalitis [12]. Die primäre virale Pneumonie, die innerhalb weniger Tage zum Tode führen kann, tritt nicht nur bei Immungeschwächten, sondern auch bei jüngeren Erwachsenen und Kindern auf. Eine sekundäre bakterielle Pneumonie ist die am häufigsten auftretende Komplikation. Die Epithelien der Atmungsorgane sind durch die Influenzaviren zum Teil schwer geschädigt, so dass es sehr leicht zu einer sekundären bakteriellen Infektion kommen kann. Die überwiegende Zahl der während einer Influenzawelle in interpandemischen Perioden registrierten Hospitalisierungen und Todesfälle entfällt auf die so genannten Risikogruppen, zu denen die Älteren und chronisch Kranken zählen [13].

\section{Auswirkungen der Influenza}

Die Auswirkungen der Influenza, d. h. der meist während der Monate Januar bis März ablaufenden Grippewelle, sind nur indirekt messbar. Wenn auch seit der Verabschiedung des Infektionsschutzgesetzes (IfSG) im Januar 2001 jeder direkte Nachweis einer Influenzavirusinfektion meldepflichtig ist, so kann nicht und braucht auch nicht für jeden Patienten mit Verdacht auf eine Influenza eine Diagnostik durchgeführt werden. Maßstab für die Einschätzung der Schwere einer Influenzaepidemie wird auch zukünftig sein, die Exzessmortalität, d. h. die Übersterblichkeit, während der Wochen und Monate mit nachgewiesener Influenzavirus-Zirkulation zu ermitteln [14]. Basis dafür ist ein gut ausgebautes Influenza-Surveillancesystem, wie es z. B. seit 1992 in enger Zusammenarbeit zwischen der Arbeitsgemeinschaft Influenza und dem Nationalen Referenzzentrum für Influenza besteht.

Übersterblichkeit bedeutet die Ermittlung der Sterblichkeitsrate während einer Zeit mit nachgewiesener Influenzavirus-Zirkulation im Vergleich zu einer über mehrere Jahre ermittelten Basislinie. In den USA werden kontinuierlich über eine jede Saison bzw. das ganze Jahr die aus den einzelnen Bundesstaaten gemeldeten Fälle unter der Rubrik „Pneumonie und Influenza“ erfasst. Die Zahlen schwanken je nach Schwere der jährlichen Influenzaepidemie. Eine Vielzahl von Studien hat gezeigt, dass im Durchschnitt in den USA jährlich etwa 20.000 Personen an der Influenza oder deren Folgen sterben $[15,16]$.

\section{Globale Surveillance}

Die ständig ablaufende Antigendrift und die Gefahr einer Antigenshift haben die Weltgesundheitsorganisation (WHO) schon 1948 veranlasst, ein globales Netz zur Überwachung der Influenza aufzubauen. In diesem Netzwerk werden sowohl epidemiologische als auch virolo-

\section{„Um eine rasche Identifizierung neuer Influenzavarianten zu ermöglichen, hat die WHO ein globales Überwachungsnetz etabliert."}

Jedes nationale Influenzazentrum meldet insbesondere während der Influenzasaison die Anzahl der nachgewiesenen Influenzaviren, deren Typ und Subtyp sowie die Beurteilung der epidemiologischen Situation. In der nördlichen Hemisphäre erstreckt sich die Influenzasaison vom Oktober bis zum April des folgenden Jahres. Die Stärke der Influenzaepidemie variiert von Jahr zu Jahr. Von Oktober bis Dezember tritt die Influenza oft nur sporadisch auf, die eigentliche Epidemie wird meist zwischen Januar und März registriert. Die Influenzasaison auf der Südhalbkugel ist weitaus vielgestaltiger. In den gemäßigten Klimazonen der südlichen Hemisphäre liegt der Gipfel der Influenzasaison vorwiegend zwischen Juni und September.

Am Beispiel zweier ausgewählter Regionen, Australien und Südamerika, soll der Verlauf der Influenzasaison in Ländern der südlichen Hemisphäre dargestellt werden. Abbildung 1 zeigt die Influenzaaktivität während des Jahres 2000 in Australien [17]. Bis zum Juli war eine sporadische Zirkulation von Influenzaviren nachweisbar. Eine starke Influenzaaktivität wurde dagegen während der Monate August und September 
Tabelle 1

Analyse der Influenzasaison: Zeitraum 1995/1996 - 2000/2001

\begin{tabular}{|c|c|c|c|c|c|c|}
\hline Saison & $\begin{array}{l}\text { Typ/ } \\
\text { Subtyp }\end{array}$ & $\begin{array}{l}\text { Isolate } \\
\text { [\%] }\end{array}$ & $\begin{array}{l}\text { Morbidität } \\
\text { [Mio] }\end{array}$ & $\begin{array}{l}\text { Arbeits } \\
\text { unfähigkeit } \\
\text { [Mio] }\end{array}$ & $\begin{array}{l}\text { Exzess- } \\
\text { mortalität }\end{array}$ & Schweregrad \\
\hline $1995 / 96$ & $\begin{array}{l}A / H 3 N 2 \\
A / H 1 N 1\end{array}$ & $\begin{array}{l}55 \\
42\end{array}$ & $8-9$ & 5 & 37.000 & $\begin{array}{l}\text { Mittelschwere } \\
\text { Epidemie }\end{array}$ \\
\hline 1996/97 & $\begin{array}{l}\text { A/H3N2 } \\
B\end{array}$ & $\begin{array}{l}43 \\
54\end{array}$ & 5 & 2,5 & $\begin{array}{l}\text { Nicht } \\
\text { signifikant }\end{array}$ & $\begin{array}{l}\text { Normale } \\
\text { Influenzawelle }\end{array}$ \\
\hline 1997/98 & $\begin{array}{l}A / H 3 N 2 \\
A / H 1 N 1\end{array}$ & $\begin{array}{r}92 \\
7\end{array}$ & 4 & 2,3 & $\begin{array}{l}\text { Nicht } \\
\text { signifikant }\end{array}$ & $\begin{array}{l}\text { Normale } \\
\text { Influenzawelle }\end{array}$ \\
\hline 1998/99 & $\begin{array}{l}A / H 3 N 2 \\
B\end{array}$ & $\begin{array}{l}67 \\
33\end{array}$ & $7-8$ & $4-5$ & 15.000 & $\begin{array}{l}\text { Ausgeprägte } \\
\text { Influenzawelle }\end{array}$ \\
\hline 1999/00 & $\begin{array}{l}\mathrm{A} / \mathrm{H} 3 \mathrm{~N} 2 \\
\mathrm{~A} / \mathrm{H} 1 \mathrm{~N} 1\end{array}$ & $\begin{array}{r}99 \\
1\end{array}$ & $4-5$ & 3,5 & n.e.* & $\begin{array}{l}\text { Normale } \\
\text { Influenzawelle }\end{array}$ \\
\hline $2000 / 01$ & $\begin{array}{l}A / H 1 N 1 \\
B\end{array}$ & $\begin{array}{r}96 \\
4\end{array}$ & $2-2,5$ & 1,5 & n.e. & $\begin{array}{l}\text { Normale } \\
\text { Influenzawelle }\end{array}$ \\
\hline
\end{tabular}

*n.e.: Nicht ermittelt, da die Daten vom Statistischen Bundesamt noch nicht vorliegen. Die Angaben zur Morbidität, Arbeitsunfähigkeit und Exzessmortalität wurden den jährlichen Abschlussberichten der AGI entnommen. Der prozentuale Anteil der in der jeweiligen Saison zirkulierenden Influenzavirustypen und -subtypen wurde vom NRZ Influenza ermittelt. registriert. Die regionalen bis weit verbreiteten Ausbrüche wurden überwiegend von Influenza-A-Viren des Subtyps $\mathrm{H}_{3} \mathrm{~N}_{2}$ verursacht. Influenza-B-Viren wurden in wesentlich geringerem Umfang isoliert. Den Verlauf der Influenzasaison in einem südamerikanischen Land zeigt Abb. 2. In Argentinien wurde seit März des Jahres 2000 ein sporadisches Auftreten der Influenza beobachtet. Die höchste Influenzaaktivität wurde im Juni und Juli registriert. Auch hier wurde die Erkrankungswelle überwiegend durch Influenza-A-Viren verursacht. InfluenzaB-Viren spielten hier ebenfalls nur eine sehr untergeordnete Rolle [17]. In einigen asiatischen Ländern werden Influenzaviren fast das ganze Jahr über nachgewiesen [20]. Eine bisaisonale Influenzaaktivität wird besonders in Südostasien, so z. B. auf den Philippinen, in Thailand, Vietnam und Südostchina, beobachtet. In Regionen mit zweifacher Influenzasaison wird eine stärkere Influenzawelle während der Monate Juni bis September registriert. Der zweite Peak ist meist wesentlich niedriger und tritt während der Monate November bis Januar auf. In tropischen Regionen sind Influenzaausbrüche nicht mit bestimmten Jahreszeiten assoziiert, Epidemien können ständig während eines Jahres auftreten.

\section{Nationale Surveillance}

Verantwortlich für die Influenzaüberwachung in Deutschland sind das Nationale Referenzzentrum (NRZ) für Influenza mit den Standorten Berlin (Robert Koch-Institut) und Hannover (Niedersächsisches Landesgesundheitsamt) sowie die Arbeitsgemeinschaft Influenza (AGI) in Marburg. Durch die AGI wurde
1992 ein Sentinelsystem zur Erfassung akuter respiratorischer Erkrankungen etabliert [21, 22]. Eine enge Zusammenarbeit zwischen dem NRZ für Influenza und der AGI bildet die Basis für eine flächendeckende virologische Surveillance. Zukünftig werden sowohl die klinische als auch die virologische Überwachung noch weiter intensiviert. Dies wird ermöglicht durch eine enge Kooperation der Surveillancepartner innerhalb der nun seit dem Jahr 2001 am RKI angesiedelten AGI. In die epidemiologische Auswertung werden zukünftig auch die über das Infektionsschutzgesetz verfügbaren Daten zum Nachweis von Influenzavirusinfektionen einfließen. Somit gehört Deutschland seit 1992 zu den Ländern mit einem flächendeckenden Netz zur Influenzaüberwachung. Es gibt jedoch in Europa Staaten wie Frankreich [23], Großbritannien [24], Belgien [25] und die Niederlande [26], die schon seit vielen Jahren über ein nationales Surveillancesystem verfügen. Ziel derartiger Überwachungssysteme ist es, den Beginn und Verlauf der Influenzaepidemie zu verfolgen sowie deren Einfluss auf das Krankheitsgeschehen zu analysieren, Gesundheitsbehörden und die Öffentlichkeit zu informieren und Einfluss darauf zu nehmen, dass die Impfung als wichtigste Prophylaxemöglichkeit noch besser genutzt wird.

In den letzten Jahren war die Influenzasaison in Deutschland recht unterschiedlich ausgeprägt. In einigen Län-

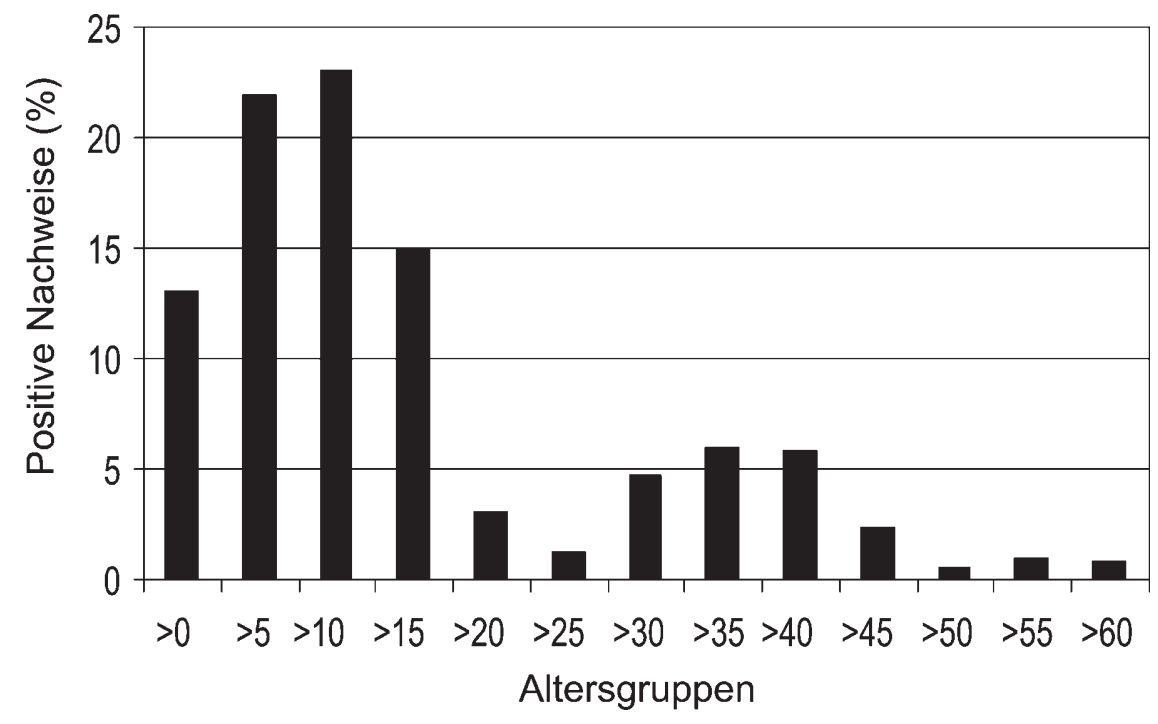

Abb. $3 \Delta$ Anteil der positiven Influenzavirusnachweise pro Altersgruppe an der Gesamtzahl der Einsendungen, die während der Saison 2000/2001 vom NRZ Influenza am Robert Koch-Institut untersucht wurden 
dern wie z. B. Frankreich wird - im epidemiologischen Sinne korrekt - jeder Zeitraum mit einem gehäuften Auftreten der Influenza als Epidemie bezeichnet. Schwierig dabei ist, die jeweiligen Unterschiede der Ausprägung des epidemischen Prozesses zu verdeutlichen. In Deutschland haben die für die Influenzasurveillance Verantwortlichen daher miteinander vereinbart, die in jedem Jahr registrierte Influenzaaktivität nicht generell als Influenzaepidemie einzustufen, sondern den Begriff der „Epidemie“ für Prozesse mit ungewöhnlich vielen Erkrankungen und schweren Verlaufsformen zu reservieren. Das hat den Vorteil, in einer dann als „Epidemie“ deklarierten Situation tatsächlich die erforderliche Aufmerksamkeit zu gewinnen und Akzeptanz für verschiedene dann zu empfehlende Maßnahmen zu erreichen. In den Jahren mit geringerer Influenzaaktivität wird eine Influenza-bedingte Erkrankungswelle konstatiert und situationsgerecht interpretiert. Das im Folgenden als „normale“ Influenzawelle bezeichnete Geschehen entspricht daher einer „leichten“ Influenzaepidemie im streng epidemiologischen Sinn.

In Tabelle 1 sind die wichtigsten Charakteristika der Influenzasaison der letzten sechs Jahre zusammengefasst. Die Saison 1995/96 war die schwerste seit vielen Jahren. Maßstab für die Auswirkungen einer Influenzaepidemie ist vor allem die Exzessmortalität während einer Saison. Während des Winters 1995/96 wird eine Übersterblichkeit von etwa 30.000 Personen angenommen [27]. Ein wichtiger Parameter, um die Schwere der Influenzaepidemie zu beurteilen, sind auch die während der Zeit mit höchster Influenzaaktivität ermittelten, über das normale Maß für diese Jahreszeit hinausgehenden Hospitalisierungen. Diese wurden allein für Dezember 1995 und Januar 1996 auf 28.000 geschätzt. Sehr aufschlussreich ist des Weiteren die Analyse der Arbeitsunfähigkeiten, d. h. der Unfähigkeit, den Verpflichtungen im Beruf, in der Schule oder im Privatleben nachzukommen. So wurden für die Saison 1995/96 etwa 7,8 Mio. Atemwegserkrankungen über das für diese Jahreszeit zu erwartende Maß hinaus angenommen. Szucs und Mitarbeiter [28] beschreiben weitere Faktoren wie Kosten für ärztliche Behandlung, Medikamente, Arbeitszeitausfall und Hospitalisierung. Im Gegensatz zur Saison 1995/96 waren die nächs- ten beiden Winter durch eine „normale“ Influenzawelle geprägt.

Während der Saison 1998/99 kam es wieder zu einer sehr starken Influenzavirusaktivität. Die Übersterblichkeit während dieser Saison wird auf ca.15.00o Personen geschätzt, die direkt oder an den Folgen der Influenza verstorben sind. Die Analyse aller Daten zur Exzessmortalität zeigt, dass überwiegend ältere Menschen und Personen mit Grunderkrankungen während der Monate mit hoher Influenzaaktivität verstorben sind [16].

\section{„Im Winter 2000/2001 erfasste die Influenza überwiegend Kinder und Jugendliche."}

Nach der durch eine recht hohe Morbidität und Mortalität gekennzeichneten Saison 1998/99 folgten zwei Winter mit „normaler“ Influenzaaktivität. Etwas ungewöhnlich war die Influenzawelle, die während der Saison 2000/2001 über Deutschland rollte. Im Sentinelsystem der AGI wurden so hohe Erkrankungszahlen bei Klein- und Schulkindern registriert, wie sie in den neun Jahren zuvor nicht erreicht wurden. Insgesamt entfielen etwa $70 \%$ aller positiven Nachweise auf die Altersgruppe der Kinder und Jugendlichen (Abb.3). Die Übereinstimmung zwischen den Ergebnissen der klinischen Surveillance, d. h. der Erfassung von akuten Atemwegserkrankungen (ARE) nach Altersgruppen, und der virologischen Surveillance [29] zeigt, dass die Influenza im Winter 2000/2001 überwiegend die Kinder und Jugendlichen erfasst hat. Die Erkrankungen wurden zu über 90\% durch Influenza-A-Viren des Subtyps $\mathrm{H}_{1} \mathrm{~N}_{1}$ hervorgerufen. Erwachsene waren nur geringfügig von

Tabelle 2

Impfstoffempfehlung der WHO seit 1985

\begin{tabular}{|c|c|c|c|}
\hline Saison & Subtyp H1N1 & Subtyp H3N2 & Influenza B \\
\hline \multicolumn{4}{|c|}{ Globale Impfstoffempfehlung } \\
\hline 1985/86 & $\mathrm{A} / \mathrm{Chile} / 1 / 83$ & A/Philippines/2/82 & B/USSR/100/83 \\
\hline 1986/87 & A/Chile/1/83 & A/Mississippi/1/85 & B/Ann Arbor/1/86 \\
\hline 1987/88 & $\mathrm{A} /$ Singapore/6/86 & A/Leningrad/360/86 & B/Ann Arbor/1/86 \\
\hline 1988/89 & A/Singapore/6/86 & A/Sichuan/2/87 & B/Beijing/1/87 \\
\hline 1989/90 & A/Singapore/6/86 & A/Shanghai/11/87 & B/Yamagata/16/88 \\
\hline 1990/91 & A/Singapore/6/86 & A/Guizhou/54/89 & B/Yamagata/16/88 \\
\hline 1991/92 & A/Singapore/6/86 & A/Beijing/353/89 & $\begin{array}{l}\mathrm{B} / \text { Yamagata/ } 16 / 88 \text { oder } \\
\mathrm{B} / \text { Panama/45/90 }\end{array}$ \\
\hline 1992/93 & A/Singapore/6/86 & A/Beijing/353/89 & $\begin{array}{l}\mathrm{B} / \text { Yamagata/ } 16 / 88 \text { oder } \\
\mathrm{B} / \text { Panama/45/90 }\end{array}$ \\
\hline 1993/94 & A/Singapore/6/86 & A/Beijing/32/92 & B/Panama/45/90 \\
\hline 1994/95 & A/Singapore/6/86 & A/Shangdong/9/93 & B/Panama/45/90 \\
\hline 1995/96 & A/Singapore/6/86 & A/Johannesburg/33/94 & B/Panama/45/90 \\
\hline 1996/97 & A/Singapore/6/86 & A/Wuhan/359/95 & B/Beijing/184/93 \\
\hline 1997/98 & A/Bayern/7/95 & A/Wuhan/359/95 & B/Beijing/184/93 \\
\hline \multicolumn{4}{|c|}{ Impfstoffempfehlung für die nördliche und südliche Hemisphäre nach Einführung der Trennung } \\
\hline 1998/99 (NH) $)^{*}$ & A/Beijing/262/95 & A/Sydney/5/97 & B/Beijing/184/93 \\
\hline $1999(\mathrm{SH})^{* *}$ & A/Beijing/262/95 & A/Sydney/5/97 & B/Beijing/184/93 \\
\hline 1999/00 (NH) & A/Beijing/262/95 & A/Sydney/5/97 & $\begin{array}{l}\text { B/Beijing/184/93 oder } \\
\text { B/Yamanashi/166/98 }\end{array}$ \\
\hline 2000 (SH) & A/New Caledonia/20/99 & A/Moscow/10/99 & $\begin{array}{l}\text { B/Beijing/184/93 oder } \\
\text { B/Shangdong/7/97 }\end{array}$ \\
\hline 2001/01 (NH) & A/New Caledonia/20/99 & A/Moscow/10/99 & $\begin{array}{l}\text { B/Beijing/184/93 oder } \\
\text { B/Yamanashi/166/98 }\end{array}$ \\
\hline 2001 (SH) & A/New Caledonia/20/99 & A/Moscow/10/99 & B/Sichuan/379/99 \\
\hline 2001/02 (NH) & A/New Caledonia/20/99 & A/Moscow/10/99 & B/Sichuan/379/99 \\
\hline
\end{tabular}

*NH: nördliche Hemisphäre, **SH: südliche Hemisphäre. 
der Influenzawelle betroffen. Da die Pädiater mit $20 \%$ der Sentinelärzte in der AGI überrepräsentiert sind, könnte allein die Erfassung von ARE/Altersgruppe/Praxis zu einer epidemiologisch nicht korrekten Aussage führen. Darüber hinaus wurde jedoch unabhängig von der Anzahl der Pädiater eine Konsultationsinzidenz pro Altersgruppe für Allgemeinmediziner und Pädiater pro 100.00o Bevölkerung ermittelt. Die Einschätzung des Ausmaßes der von der Influenzawelle im letzten Winter betroffenen Altersgruppen erfolgte daher aufgrund epidemiologisch fundierter Analysen. Eine signifikante Übersterblichkeit ist für die Saison 2000/2001 nicht zu erwarten, da die besonders gefährdeten älteren Menschen von der Welle kaum erfasst waren. Eine exakte Analyse der Exzessmortalität kann erst vorgenommen werden, wenn die Daten des Statistischen Bundesamtes zur Verfügung stehen. Eine erhöhte Hospitalisierungsrate wurde für die Kleinkinder (0-4 Jahre), aber auch die älteren Menschen ( $>60$ Jahre) registriert. Für beide Altersgruppen können etwa 5000 Hospitalisierungen über das normale Maß hinaus geschätzt werden (s. Beitrag von Uphoff et al. in diesem Heft [29]).

\section{Jährliche Impfstoffempfehlung}

Jedes der 110 nationalen Influenzazentren, die mit der WHO zusammenarbeiten, führt insbesondere während der Influenzasaison eine intensive Überwachung durch. Dies bedeutet für Länder der südlichen Hemisphäre zum Teil eine ganzjährige und für die Länder der nördlichen Hemisphäre eine Überwachung vom Herbst bis zum Frühjahr des kommenden Jahres. Surveillance während der jeweiligen Saison umfasst vor allem Isolierung, Typisierung, Subtypisierung und Charakterisierung der zirkulierenden Influenzaviren. $\mathrm{Da}$ die $\mathrm{Vi}$ rusanzucht sich über zwei bis drei Wochen erstrecken kann, setzen viele Labore eine Schnelldiagnostik ein, um die epidemiologische Situation sehr zeitnah beurteilen zu können. Hier steht eine recht breite Palette von Methoden wie Immunfluoreszenz [30], Enzymimmunoassays [31] bis hin zu molekularbiologischen Verfahren [32] zur Verfügung, die sich allerdings hinsichtlich ihrer Sensitivität unterscheiden. Diese Methoden gestatten, dass wenige Stunden nach Eingang des Patientenmaterials schon das erste Resultat vorliegt. Wesentliche Vorteile derartiger Schnelldiagnostik bestehen darin, dass Beginn, Ausdehnung und auch Abklingen der Influenzawelle stets aktuell verfolgt werden können. Eine Differenzierung der nachgewiesenen Influenzaviren ist durch den Einsatz geeigneter Schnellteste ebenfalls gegeben. So kann eingeschätzt werden, ob und in welchem Ausmaß Influenza-A- oder Influenza-B-Viren zirkulieren bzw. ob innerhalb der Influenza-A-Viren der Subtyp $\mathrm{H}_{3} \mathrm{~N}_{2}$ oder $\mathrm{H}_{1} \mathrm{~N}_{1}$ dominiert. Informationen über den Verlauf der Influenzawelle, Anzahl sowie Typ und Subtyp der nachgewiesenen Influenzavirusinfektionen werden im Rahmen des FluNet der WHO zur Verfügung gestellt.

Um die Verwandtschaft der während einer Saison zirkulierenden Influenzaviren mit den Impfstämmen oder den Viren, die im vorherigen Jahr die Influenzawelle verursachten, zu untersuchen, müssen die isolierten Viren umfassend charakterisiert werden. Dazu wird von allen Zentren der Hämagglutinationshemmtest [33] eingesetzt. In diesem Test werden die während der Saison isolierten Viren kontinuierlich mit einem definierten Panel spezifischer Immunseren untersucht. Eine repräsentative Auswahl der isolierten Viren wird mehrmals im Laufe der Saison der WHO zu vergleichenden Untersuchungen zur Verfügung gestellt. Ausgewählt werden von den Influenzazentren vor allem Stämme, die das nationale Geschehen sehr gut repräsentieren. Ebenso wichtig ist es aber auch, solche Viren an die
WHO zu senden, die sich von der Mehrzahl der zirkulierenden Stämme unterscheiden. Nur so besteht die Möglichkeit, das Auftreten und die beginnende Verbreitung von neuen Viren mit veränderten Eigenschaften zu erfassen.

\section{„Die Daten der nationalen und globalen Influenzaüber- wachung sind Basis der jähr- lichen Impfstoffempfehlung."}

Die vier regionalen WHO-Zentren in Atlanta, London, Melbourne und Tokio vergleichen die Stämme der nationalen Zentren mit Viren, die in anderen Regionen der Erde isoliert wurden. Diese regionalen Zentren können somit Auskunft darüber geben, welche Virusvarianten in Europa, den USA oder auf der Südhalbkugel zirkulieren. Gemeinsam wird in Rahmen einer Expertenkommission evaluiert, welche Varianten am stärksten verbreitet sind und mit hoher Wahrscheinlichkeit während der nächsten Influenzasaison zirkulieren werden. Aufgrund dieser Evaluierung wird dann die Impfstoffempfehlung für die kommende Saison herausgegeben. Diese wird in der Regel von regionalen oder nationalen Gremien noch einmal vor dem Hintergrund von regionalen und/oder nationalen Gegebenheiten evaluiert bzw. als Empfehlung bestätigt. In Europa übernimmt diese Aufgabe die European Agency for the Evaluation of Medicinal Products (EMEA, Europäische Agentur zur Beurteilung von Arzneimitteln). Maßgebliches Gremium für die Annah-
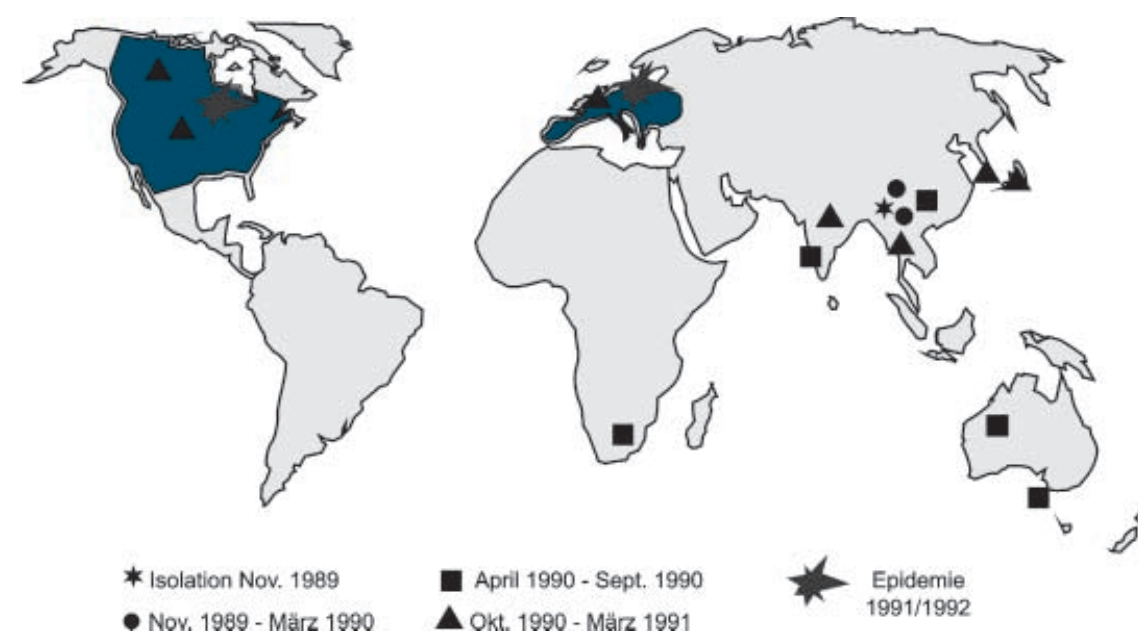

April 1990 - Sept. 1990 A Okt. 1990 - März 1991

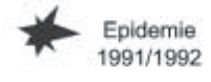

Abb. 4 A Ausbreitung von Beijing/353/89-like-Viren (Quelle: WHO, Weekly Epidemiological Record $65: 53-56,1990 ; 66: 57-60,1991)$ 
me der WHO-Empfehlung ist hier das Committee for Proprietary Medicinal Products (CPMP, Ausschuss für Arzneimittelspezialitäten). Die Impfstoffempfehlung für Europa wird durch den CPMP nach Evaluierung der Daten zur europäischen Influenzaüberwachung, wofür die Ad hoc working group on influenza vaccines zuständig ist, bekannt gegeben [34].

Der Influenzavirusimpfstoff ist ein Drei-Komponentenimpfstoff. Enthalten sind die Influenzavirus-Typen, die generell jedes Jahr zu erwarten sind, d. h. der Impfstoff besteht aus einer aktuellen Variante von Influenza-A-Viren des Subtyps $\mathrm{H}_{1} \mathrm{~N}_{1}$, des Influenza-A-Subtyps $\mathrm{H}_{3} \mathrm{~N}_{2}$ und des Influenzavirus Typ B. Ob ein definierter Stamm ein, zwei, drei Jahre oder eventuell auch länger als Impfvirus empfohlen wird, ist abhängig von der globalen Situation. Tabelle 2 informiert darüber, welche Stämme seit 1985 in der jährlichen Impfstoffempfehlung enthalten waren. Diese globale Empfehlung galt bis zum Jahr 1998. Seit dieser Zeit wird eine getrennte Empfehlung für die südliche und die nördliche Hemisphäre ausgesprochen. Ein Vergleich der einzelnen Impfstoffkomponenten zeigt, dass einige Stämme über einen relativ langen Zeitraum im Impfstoff verblieben. Der Stamm A/Singapore/6/86 wurde ein Jahr nach seiner Isolierung als $\mathrm{H}_{1} \mathrm{~N}_{1}-$ Komponente empfohlen und diente als Impfstamm bis zur Saison 1996/97. Influenza-A-Viren vom Subtyp $\mathrm{H}_{1} \mathrm{~N}_{1}$ sind weitaus weniger variabel als Subtyp$\mathrm{H}_{3} \mathrm{~N}_{2}$-Viren und Influenza-B-Viren. Viren vom Subtyp $\mathrm{H}_{1} \mathrm{~N}_{1}$ sind in der Regel auch in geringerem Umfang an Influenzaausbrüchen und Epidemien beteiligt (vgl. Tabelle 1). Der Vergleich der Impfstämme über einen längeren Zeitraum zeigt außerdem, dass auch einige Typ-BStämme über mehrere Jahre im Impfstoff enthalten waren. Als Beispiel sei hier der Stamm B/Panama/45/90 aufgeführt, der ein Jahr nach seiner Isolierung als Impfstoffkomponente für die Saison 1991/92 vorgeschlagen und erst in der Saison 1996/97 durch den Stamm B/Beijing/184/93 abgelöst wurde. Neue Driftvarianten von Influenza B sind auch nicht in einer so hohen Frequenz nachweisbar wie bei Influenza-A-Viren des Subtyps $\mathrm{H}_{3} \mathrm{~N}_{2}$. Die $\mathrm{H}_{3} \mathrm{~N}_{2}$-Komponente im Impfstoff variierte sehr stark (s. Tabelle 2). Keiner der in den letzten 15 Jahren empfohlenen $\mathrm{H}_{3} \mathrm{~N}_{2}$-Impfstämme

Tabelle 3

Serologische Charakterisierung der während der Saison 1997/1998 zirkulierenden Influenzaviren des Subtyps H3N2

\begin{tabular}{|c|c|c|c|c|}
\hline \multirow[t]{2}{*}{ Stamm } & \multicolumn{4}{|c|}{ Immunserum } \\
\hline & $\begin{array}{l}\text { A/Wuhan/ } \\
359 / 95\end{array}$ & $\begin{array}{l}\text { A/Nanchang/ } \\
\text { 933/95 }\end{array}$ & $\begin{array}{l}\text { A/Sydney/ } \\
5 / 97\end{array}$ & $\begin{array}{l}\text { A/Saga/ } \\
128 / 97\end{array}$ \\
\hline A/Wuhan/359/95 & 640 & 320 & 40 & 80 \\
\hline A/Nanchang/933/95 & 640 & 640 & 80 & 160 \\
\hline A/Sydney/5/97 & 80 & 160 & 1280 & 1280 \\
\hline A/Saga/128/97 & 160 & 320 & 1280 & 1280 \\
\hline A/Hong Kong/544/97 & 640 & 640 & 160 & 160 \\
\hline A/Netherlands/4/98 & 640 & 1280 & 160 & 160 \\
\hline A/Oslo/96/98 & 640 & 1280 & 80 & 160 \\
\hline A/Texas/1482/97 & 640 & 640 & 160 & 160 \\
\hline A/Hong Kong/541/97 & 160 & 160 & 1280 & 1280 \\
\hline A/Malmö/3/98 & 160 & 320 & 2560 & 2560 \\
\hline A/Parma/1/98 & 160 & 320 & 1280 & 1280 \\
\hline A/Shiga/25/97 & 160 & 320 & 1280 & 2560 \\
\hline A/Washington/508/97 & 160 & 160 & 1280 & 1280 \\
\hline \multicolumn{5}{|c|}{$\begin{array}{l}\text { In der Tabelle sind die von regionalen WHO-Zentren im Hämagglutinationshemmtest (HHT) ermittelten } \\
\text { reziproken Titer aufgeführt (Quelle: WHO: Weekly Epidemiological Record 73:56-62, 1998). Je geringer } \\
\text { die Titerdifferenz im HHT zwischen einem Isolat und einem Impfstamm, desto größer ist die serologische } \\
\text { Verwandtschaft. Beispiel: Der Stamm A/Oslo/96/98 reagierte sehr schlecht mit dem Immunserum } \\
\text { gegen den Referenzstamm A/Sydney/5/97 (Titer 1:80), aber sehr gut mit dem Immunserum gegen } \\
\text { A/Wuhan/359/95 (Titer 1:640) und wurde daher als Wuhan-like eingestuft. }\end{array}$} \\
\hline
\end{tabular}

verblieb länger als zwei Jahre im Impfstoff. Während dieser Zeit wurden dann wieder das Auftreten und die Verbreitung von Viren mit signifikant verändertem Antigenprofil beobachtet. Solche Driftvarianten machten es erforderlich, die Zusammensetzung des Impfstoffes stets zu aktualisieren.

Die Gegenüberstellung der Impfstoffstämme zeigt auch deutlich, dass die $\mathrm{H}_{3} \mathrm{~N}_{2}$-Komponente überwiegend im Jahr nach Auftauchen einer neuen Driftvariante ausgetauscht wurde. Auch wenn Viren mit veränderten antigenen Eigenschaften in einer Region nachgewiesen werden, so bedeutet dies nicht zwangsläufig, daran die Impfstoffempfehlung auszurichten. Als Beispiel sei hier die Ausbreitung von A/Beijing/353/89-like-Viren genannt (Abb. 4), die im November 1989 zum ersten Mal in Peking isoliert wurden. Innerhalb der nächsten vier Monate wurden Viren mit einem solchen Profil ausschließlich in Südostchina nachgewiesen. Während der dann beginnenden Saison auf der Südhalbkugel fand man Beijing/353/89-like-Viren weiterhin in Südostchina, aber auch in Indien, Australien und Südafrika. Ab Oktober 1990 war die- se Driftvariante auch auf der Nordhalbkugel nachweisbar, denn sie verursachte kleinere Ausbrüche in den USA und Europa. Die nun mittlerweile globale Verbreitung dieser neuen Driftvariante veranlasste die WHO, den Stamm A/Beijing/353/89 als $\mathrm{H}_{3} \mathrm{~N}_{2}$-Komponente für die Saison 1991/92 vorzuschlagen. Diese Empfehlung erwies sich als zutreffend, denn während des Winters 1991/92 wurden die in den USA und Europa ablaufenden Epidemien durch Beijing/353/89-likeViren hervorgerufen [35].

Dies ist ein Beispiel von vielen, dass die Impfstoffempfehlung solche Stämme enthielt, die während der folgenden Saison tatsächlich zirkulierten. Es gab aber auch Ausnahmen. Erwähnt sei hier die schnelle Verbreitung von Influenza-AViren, die 1997 zum ersten Mal in Sydney isoliert wurden. Im Winter 1995/96 zirkulierten nicht nur in Deutschland, sondern überall auf der Welt vorrangig A/Wuhan/353/95-like-Viren [36]. Deshalb verblieb dieser Stamm auch für ein weiteres Jahr im Impfstoff. Die Impfstoffempfehlung für die nördliche Hemisphäre wird jeweils im Februar herausgegeben, damit die Impfstoffhersteller die er- 
forderliche Zeit zur Produktion, Prüfung und Freigabe des Impfstoffes zur Verfügung haben. Im August 1997, als der Impfstoff für die kommende Saison bereits produziert war, wurde ein Virus isoliert, das sich beträchtlich von den Wuhan-like-Viren unterschied. Dieser Stamm A/Sydney/5/97 erfüllte die Kriterien der „klassischen“ Definition für eine neue Driftvariante mit einer Titerdifferenz von 4 im Hämagglutinationshemmtest. In Tabelle 3 sind einige Virusstämme gegenübergestellt, die während der Saison 1997/98 in verschiedenen Erdteilen isoliert wurden [37]. Unter ihnen waren sowohl Wuhan/ 359/95- als auch Sydney/5/97-like-Stämme zu finden. Ein
Vertreter der Wuhan-like-Viren war z. B. der Stamm A/Oslo/96/98, wohingegen das Isolat A/Malmö/3/98 als Sydney-like charakterisiert wurde. Ein vergleichbares Bild zeigte sich auch in Europa und Deutschland. Die ersten Viren der Saison wurden noch als Wuhan-like klassifiziert. Diese Viren wurden aber sehr schnell von der neuen Driftvariante Sydney/5/97 verdrängt und nur noch vereinzelt nachgewiesen. Obwohl die neue Driftvariante Sydney/5/97 nicht Bestandteil des Impfstoffes war, kam es während der Saison 1997/98 dennoch zu keiner schweren Influenzawelle [38]. Die vorrangig zirkulierenden Sydney/5/97-likeViren reagierten zwar weniger gut mit dem Immunserum gegen den $\mathrm{Wu}-$ han/359/95-Stamm, wurden jedoch in gewissem Umfang durch Antikörper erkannt. Dies erklärt auch, dass es zu keinen größeren Impfdurchbrüchen kam.

Für den Impfstoff der Saison 2001/o2 wurden folgende Stämme vorgeschlagen:

D A/New Caledonia/20/99

(Typ A, Subtyp H1N1),

- A/Moscow/10/99 (Typ A, Subtyp

$\left.\mathrm{H}_{3} \mathrm{~N}_{2}\right)$,

D B/Sichuan/379/99 (Typ B).

Die H1N1- und die $\mathrm{H}_{3} \mathrm{~N}_{2}-$ Komponente waren bereits im vergangenen Jahr im Impfstoff vertreten. Ausgetauscht wurde

Tabelle 4
Serologische Charakterisierung der während der Saison 2000/2001 in Deutschland zirkulierenden Influenza-A-Viren
des Subtyps H1N1

Stamm

Immunserum

\begin{tabular}{llllll}
\hline A/Bayern/ & A/Johannesburg/ & A/Beijing/ & A/New Caledonia/ & A/Hongkong/ & A/Madagascar/ \\
$7 / 95$ & $82 / 96$ & $262 / 95$ & $20 / 99$ & $1252 / 00$ & $57794 / 00$
\end{tabular}

\section{Referenzstämme}

A/Bayern/7/95

A/Johannesburg/82/96

A/Beijing/262/95

A/New Caledonia/20/99

A/Hongkong/1252/00

A/Madagascar/5794/00

$\begin{array}{cc}2560 & 5120 \\ 2560 & 5120 \\ 160 & 160 \\ 80 & 80 \\ < & < \\ 80 & 80\end{array}$

160

$\begin{array}{cr}160 & 160 \\ 2560 & 640 \\ 160 & 1280 \\ < & 160 \\ 320 & 1280\end{array}$

Isolate aus Deutschland

A/Hannover/142/00

A/Hannover/144/00

A/Hannover/1/01

A/Hannover/25/01

A/Bayern/1/01

A/Bayern/5/01

A/Berlin/133/00

A/Berlin/136/00

A/Berlin/144/00

A/Berlin/146/00

A/Berlin/17/01

A/Meckl.-Vorp./4/01

A/Meckl.-Vorp./6/01

A/Meckl.-Vorp./15/01

A/Nordrh.-Westf./1/01

A/Nordrh.-Westf./9/01

A/Rheinland-Pfalz/1/01

A/Saarland/11/01

$<\quad 160$

$<\quad 160$

$<\quad 160$

$<\quad 160$

$80 \quad 160$

$40 \quad 160$

$40 \quad 80$

$40 \quad 80$

$<\quad<$

$<\quad 40$

$40 \quad 80$

$40 \quad 80$

$40 \quad 80$

$40 \quad 40$

$80 \quad 80$

$40 \quad 40$

$40 \quad 80$

$<40$

In der Tabelle sind die im Hämagglutinationshemmtest (HHT) ermittelten reziproken Titer aufgeführt. Dargestellt ist das Ergebnis der Untersuchungen ausgewählter Stämme, die in Deutschland isoliert, vom NRZ Influenza in Hannover feintypisiert und im regionalen WHO-Zentrum in London weiter analysiert wurden. Je geringer die Titerdifferenz im HHT zwischen einem Isolat und einem Impfstamm, desto größer ist die serologische Verwandtschaft. Beispiel: Der Stamm A/Berlin/144/00 reagierte sehr schlecht mit dem Immunserum gegen den Referenzstamm A/Beijing/262/95 (Titer 1:80), aber sehr gut mit dem Immunserum gegen den aktuellen Impfstamm A/New Caledonia/20/99 (Titer 1:1280) und wurde daher als New Caledonia-like eingestuft. 
der bis dahin verwendete Stamm B/ Yamanashi/166/98 durch den Stamm B/Sichuan/379/99 [39]. Dieser Stamm wurde im vergangenen September als Influenza-B-Komponente des Impfstoffes der Südhalbkugel für 2001 ausgewählt [40]. Sichuan/379/99-like-Viren wurden bereits im letzten Winter auch auf der Nordhalbkugel gefunden und aufgrund ihrer Verbreitung als Impfstamm für die jetzt beginnende Saison 2001/02 empfohlen. Die serologischen Feintypisierungen der im letzten Winter kaum zirkulierenden $\mathrm{H}_{3} \mathrm{~N}_{2}$-Viren und zu über 90\% nachgewiesenen $\mathrm{H}_{1} \mathrm{~N}_{1}-\mathrm{Vi}$ ren zeigten, dass sich bei beiden Subtypen keine signifikanten Trends erkennen ließen und somit keine Änderung dieser Komponenten im Impfstoff erforderlich war [39]. Tabelle 4 zeigt das Ergebnis der Untersuchung ausgewählter Stämme, die in Deutschland isoliert und im WHO-Zentrum in London feintypisiert wurden. In die Analyse wurden Referenzstämme, aber auch aktuelle Isolate aus Asien (A/Hongkong/1252/oo) und Südafrika (A/Madagaskar/5794/oo) einbezogen. Die in Deutschland isolierten H1N1-Viren zeigten sehr wenig Ähnlichkeit mit dem in Hongkong isolierten Stamm. Im Gegensatz dazu reagierten die deutschen Stämme sehr gut mit den Immunseren gegen den Impfstamm New Caledonia/20/99 und den auf Madagaskar isolierten Virus. Am Beispiel des Stammes Hongkong/1252/oo wird deutlich, dass hier ein Virus isoliert wurde, das auch die Kriterien für eine Driftvariante erfüllen würde. Die Untersuchungen der von den nationalen Zentren der WHO zur Verfügung gestellten Viren belegen immer wieder das Auftauchen von Influenzaviren mit z. T. signifikant veränderten serologischen Eigenschaften. Auch wenn solche Viren nur zu einem ganz geringen Prozentsatz isoliert werden, ist ihrer Ausbreitung besondere Aufmerksamkeit zu widmen. Die Mehrheit der in allen Erdteilen isolierten $\mathrm{H}_{1} \mathrm{~N}_{1}$-Viren zeigte jedoch eine sehr enge Verwandtschaft mit dem Stamm A/New/Caledonia/20/99, so dass dieser Stamm für die bevorstehende Saison wieder als Impfstamm empfohlen wurde.

\section{Literatur}

1. Murphy BR, Webster RG (1996) Orthomyxoviruses. In: Fields BN, Knipe DM, Howley PM et al. (eds) Fields virology, 3rd edn. Lippincott-Raven, Philadelphia, pp 1397-1445

2. Couch RB, Kasel JA (1983) Immunity to influenza in man. Annu Rev Microbiol 37:529-549

3. Hinshaw VS, Webster RG, Turner B (1978) Novel influenza $A$ viruses isolated from Canadian feral ducks: including strains antigenically related to swine influenza (Hsw1N1) viruses. J Gen Virol 41:115-127

4. Taubenberger JK, Reid AH, Krafft AE, Bijwaard KE, Fanning TG (1997) Initial genetic characterization of the 1918 „Spanish“ Influenza Virus. Science 275:1793-1796

5. Taubenberger JK, Reid AH, Fanning TG (2000) The 1918 influenza virus: a killer comes into view (Minireview). Virology 274:241-245

6. Scholtissek C, Rohde W, Von Hoyningen V, Rott R (1978) On the origin of the human influenza virus subtypes H2N2 and H3N2. Virology 87:13-20

7. Nakajima K, Desselberger U, Palese P (1978) Recent human influenza A (H1N1) viruses are closely related genetically to strains isolated in 1950. Nature 274:334-339

8. Nicholson KG (1992) Clinical features of influenza. Semin Respir Infect 7:26-37

9. Anonymus (1997) Tödlich verlaufende Influenza-B-Infektion. Epid Bull 8/97:53

10. Anonymus (1999) Fallbericht: Fataler Verlauf der Influenza-Erkrankung einer jungen Frau. Epid Bull 8/99:51

11. Nolte KB, Alakija P,Oty G et al. (2000) Influenza A virus infection complicated by fatal myocarditis. Am.J Forensic Med Pathol 21:375-379

12. Okabe N, Yamashita K, Taniguchi K, Inouye S (2000) Influenza surveillance system of Japan and acute encephalitis and encephalopathy in the influenza season. Pediatr Int 42: 187-191

13. Barker WH, Mullooly JP (1982) Pneumonia and influenza deaths during epidemics: implications for prevention. Arch Intern Med 142:85-89

14. Barker WH, Mullooly JP (1982) A study of excess mortality during influenza epidemics in the United States, 1968-1976. Am J Epidemiol 115:479-480

15. Lui KJ, Kendal AP (1987) Impact of influenza epidemics on mortality in the United States from October 1972 to May 1985. Am J Public Health 77:712-716

16. Simonsen L, Clarke MJ, Williamson GD, Stroup DF, Arden NH, Schonberger LB (1997) The Impact of Influenza Epidemics on Mortality: Introducing a Severity Index. Am J Public Health 87:1944-1950

17. http://oms2.b3e.jussieu.fr/flunet/index.html, http://oms2.b3e.jussieu.fr/flunet/ centres_partners.html,http://www.who.int/ emc/diseases/flu/centres.html

18. de Jong JC, Claas EC, Osterhaus ADME, Webster RG, Lim WL (1997) A pandemic warning? Nature 389:554

19. Guan Y, Shortridge KF, Krauss S, Webster RG (1999) Molecular characterization of $\mathrm{H} 9 \mathrm{~N} 2$ influenza viruses: Were they the donors of the „internal" genes of H5N1 viruses in Hong Kong? Proc Natl Acad Sci USA 96:9363-9367

20. Hampson AW (1999) Epidemiological data on influenza in Asian countries. (Summary report). Vaccine 17:S19-S23

21. Szecsenyi J, Uphoff H, Ley S, Brede HD (1995) Influenza surveillance: experiences from establishing a sentinel surveillance system in Germany.J Epidemiol Community Health 49 [Suppl 1]:9-13
22. Uphoff H (1998) A study of reasons for an increase in acute respiratory tract infections reported by influenza sentinel practices in Germany.J Epidemiol Community Health 52 [Suppl 1]:43S-45S

23. Dab W, Quenel P, Cohen JM, Hannoun C (1991) A new influenza surveillance system in France: the Ile-de-France „GROG", 2.Validity of indicators (1984-1989). Eur J Epidemiol 7:579-587

24. Fleming DM, Crombie DL, Norbury CA, Cross KW (1990) Observations on the influenza epidemic of November/December 1989. Br J Gen Pract 40:495-497

25. Snacken R, Lion J, Van Casteren V et al. (1990) Five years of sentinel surveillance of acute respiratory infections (1985-1990): the benefits of an influenza early warning system. Eur J Epidemiol 8:485-490

26. Bartelds AIM, Fracheboud J, Van der Zee J (1989) The Dutch sentinel practice network; relevance for public health policy. Studies from the continuous morbidity registration in The Netherlands. Netherlands Institute of Primary Health Care (NIVEL), Utrecht

27. Anonymus (1996) Zur Influenza-Epidemie 1995/96: Ausmaß und Folgen. Epid Bull 37/96:253-254

28. Szucs T, Behrens M,Volmer T (2001) Public health costs of influenza in Germany 1996 - a cost-ofillness analysis. Med Klin 96:63-70

29. Uphoff H,Heckler R, Schweiger B (2001) Ergebnisse der Influenza-Surveillance im Winter 2000/2001. Bundesgesundheitsbl 44:1169-1173

30. Spada B, Biehler K, Chegas P, Kaye J, RiepenhoffTalty M (1991) Comparison of rapid immunofluorescence assay to cell culture isolation for the detection of influenza $A$ and $B$ viruses in nasopharyngeal secretions from infants and children. J Virol Methods 33:305-310

31. Waner JL, Todd SJ, Shalaby H, Murphy P, Wall LV (1991) Comparison of directigen FLU-A with viral isolation and direct immunofluorescence for the rapid detection and identification of influenza $A$ virus. J.Clin Microbiol 29:479-482

32. Schweiger B, Zadow I, Heckler R, Timm H, Pauli G (2000) Application of a fluorogenic PCR assay for typing and subtyping of influenza viruses in respiratory samples.J Clin Microbiol 38:1552-1558

33. Chakraverty P (1971) Antigenic relationship between influenza $B$ viruses. Bull World Health Organ 45:755-766

34. http://www.emea.eu.int; http://www.emea.eu.int/pdfs/human/bwp/ 084000en.pdf

35. Anonymus (1991) Recommended composition of influenza virus vaccines for use in the 1991-1992 season. WER 66:57-60

36. Anonymus (1996) Recommended composition of influenza virus vaccines for use in the 1996-1997 season. WER 71:57-61

37. Anonymus (1998) Recommended composition of influenza virus vaccines for use in the 1998-1999 season. WER 73:56-62

38. Anonymus (1998) Zusammenfassende Einschätzung der Influenza-Situation 1997/98. Epid Bull 19/98:136-137

39. Anonymus (2001) Recommended composition of influenza virus vaccines for use in the 2001/2002 season. WER 76:58-61

40. Anonymus (2000) Recommended composition of influenza virus vaccines for use in 2001. WER 75:330-333 